\title{
MODIS solar diffuser degradation determination and its spectral dependency
}

Shihyan Lee, Gerhard Meister

Shihyan Lee, Gerhard Meister, "MODIS solar diffuser degradation determination and its spectral dependency," Proc. SPIE 10764, Earth Observing Systems XXIII, 1076414 (7 September 2018); doi: $10.1117 / 12.2321392$

SPIE Event: SPIE Optical Engineering + Applications, 2018, San Diego, California, United States 


\title{
MODIS solar diffuser degradation determination and its spectral dependency
}

\author{
Shihyan Lee ${ }^{1,2^{*}}$, and Gerhard Meister ${ }^{2}$ \\ Science Applications International Corporation, McLean, VA 22102; \\ NASA/GSFC/OBPG, Code 616 Greenbelt, MD 20771;
}

\begin{abstract}
This study presents a modeling approach to improve solar diffuser (SD) degradation determination from SD stability monitor (SDSM) measurements. The MODIS instrument uses a SD to calibrate its reflective solar bands (RSBs) on-orbit. Due to the imperfectly designed SDSM sun view screen, the SD reflectance tracked by SDSM has large noise. The SDSM measurements noise is spectrally coherent and can be minimized by normalizing measurements to the least degraded detector $9(936 \mathrm{~nm})$. In this study, a SD degradation model is used to determine the SD degradation's wavelength dependency and the detector 9 degradation is estimated by the model solution.

The results show the SD degradations measured at 6 SDSM detectors (554-936 nm) have stable relationships, where the degradation is inversely proportion to $1 /$ wavelength $\wedge 4$. The model estimated SD degradation at SDSM detector 9 wavelength $(936 \mathrm{~nm})$ is $\sim 0.9 \%$ from 2002 to 2018 . Based on the SD degradation model solution, the SD degradation at short/mid wave bands are estimated to improve short/mid wave bands calibration. The model can also be used to improve interpolating SD degradation at SDSM detectors to RSB wavelengths. Compared to linear interpolation, bands 9 and 10 show the largest differences of up to 0.3 and $0.4 \%$ respectively. These differences directly impact the calibration coefficients of these bands.
\end{abstract}

keywords — solar diffuser, solar diffuser stability monitor, SD/SDSM screen, degradation, radiometric calibration, MODIS, Aqua. 


\section{Introduction}

The Moderate Resolution Imaging Spectroradiometer (MODIS) on the Earth Observing System (EOS) Aqua platform has 36 spectral bands to provide near global observation every 2 days [1]. Of the 36 spectral bands, 20 are reflective solar bands (RSB) with spectral range of 0.41 to $2.1 \mu \mathrm{m}$. Within RSB, bands 8-16 are optimized to observe ocean biological processes (Table 1), and the rest of the RSB are designed for land and atmosphere applications.

Table 1. MODIS RSB specification.

\begin{tabular}{|c|c|c|}
\hline $\begin{array}{c}\text { Land/Atmos } \\
\text { phere }\end{array}$ & $\begin{array}{c}\text { Center } \\
\text { Wavelength }(\mathrm{nm})\end{array}$ & $\begin{array}{c}\text { Band Width } \\
(\mathrm{nm})\end{array}$ \\
\hline Band 1 & 645 & 50 \\
\hline Band 2 & 859 & 35 \\
\hline Band 3 & 469 & 20 \\
\hline Band 4 & 555 & 20 \\
\hline Band 5 & 1240 & 20 \\
\hline Band 6 & 1640 & 24 \\
\hline Band 7 & 2130 & 50 \\
\hline Band 17 & 905 & 30 \\
\hline Band 18 & 936 & 10 \\
\hline Band 19 & 940 & 50 \\
\hline Band 26 & 1375 & 30 \\
\hline
\end{tabular}

\begin{tabular}{|c|c|c|}
\hline Ocean & $\begin{array}{c}\text { Center } \\
\text { Wavelength }(\mathrm{nm})\end{array}$ & $\begin{array}{c}\text { Band Width } \\
(\mathrm{nm})\end{array}$ \\
\hline Band 8 & 412 & 15 \\
\hline Band 9 & 443 & 10 \\
\hline Band 10 & 488 & 10 \\
\hline Band 11 & 531 & 10 \\
\hline Band 12 & 551 & 10 \\
\hline Band 13 & 667 & 10 \\
\hline Band 14 & 678 & 10 \\
\hline Band 15 & 748 & 10 \\
\hline Band 16 & 869 & 10 \\
\hline
\end{tabular}

Table 2. MODIS SDSM detector wavelength.

\begin{tabular}{|c|c|}
\hline SDSM & Wavelength \\
\hline Detector 1 & 412 \\
\hline Detector 2 & 466 \\
\hline Detector 3 & 530 \\
\hline Detector 4 & 554 \\
\hline Detector 5 & 646 \\
\hline Detector 6 & 747 \\
\hline Detector 7 & 857 \\
\hline Detector 8 & 904 \\
\hline Detector 9 & 936 \\
\hline
\end{tabular}

The primary calibration for RSB is based on SD and SDSM assembly (Figure 1). The SD is a 
lambertian surface used as the calibration target. The SDSM is a ratioing radiometer used to track the change in SD reflectivity at 9 different wavelengths (Table 2). The SD has a removable screen to give SD two radiance levels when illuminated by the sun. The high and low SD radiance levels are designed to calibrate land and ocean bands near their typical radiances. The SDSM has a fixed screen to set the SDSM sun view radiances at similar level as SD view to reduce potential uncertainty from SDSM detector response nonlinearity.

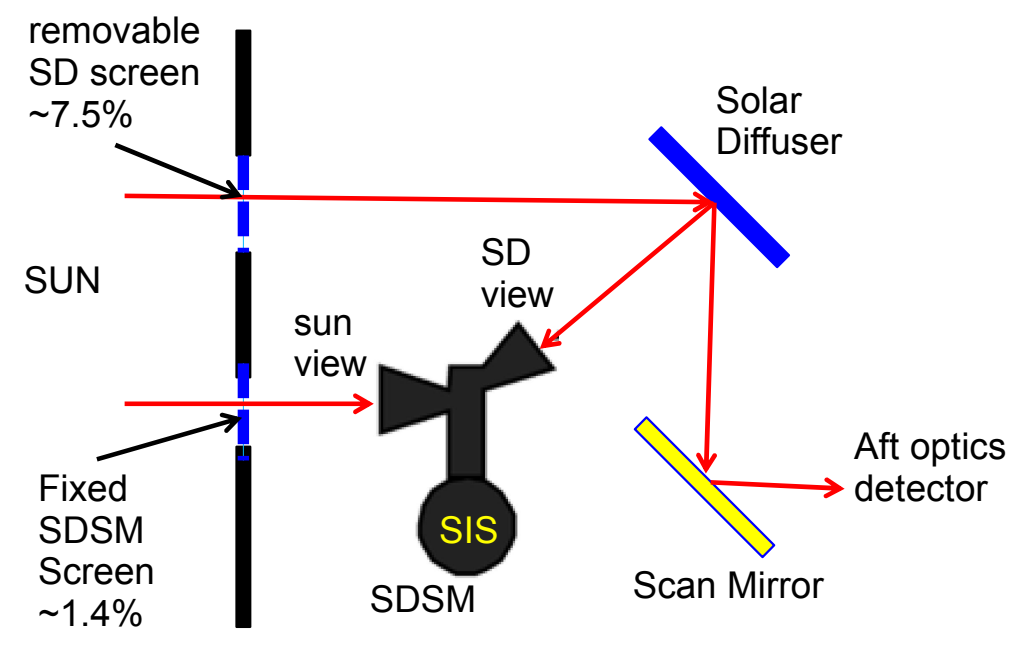

Figure 1. Schematic of SD/SDSM calibration assembly.

To compute the RSB detector's gain, we need to know the changes in both the detector's response and SD's reflectivity. The reflectivity of SD is known to degrade on-orbit. To determine the change in SD reflectivity, the SDSM sun screen's vignetting function and the SD's Bi-direction Reflectance Function (BRF) are needed. To determine the RSB detector response, the SD's BRF and its screen's vignetting function are needed. Note that SD BRF is slightly different when viewing from sensor and SDSM. The screens' vignetting function and BRF are wavelength dependent. For SD at sensor view angle, it is derived for each band. For SDSM, it is derived for each SDSM detector. The SD degradation is first estimated at SDSM detector's wavelength then interpolated to each RSB's center wavelength.

The primary cause of SD degradation is the change in surface roughness due to exposure to solar UV radiation and energetic particles [2]. The change in SD surface roughness will change the scattering characteristics and the SD BRF. Based on a multiple scattering model, the change in SD 
BRF have been shown to be inversely proportion to $1 /$ wavelength^ 4 [2]. This relationship had been confirmed by previous studies on SNPP VIIRS [2,3]. In this study, we will use the spectral degradation model to improve estimates in SD degradation for RSB calibration.

\section{SD/SDSM screens and SD BRF}

The Aqua MODIS performed yaw maneuvers at June 14, 2002 to characterize BRF of SD, and solar attenuation screens of SD and SDSM [4]. During consecutive solar calibrations, the spacecraft is yawed at designed angle to create an evenly space yaw angle to cover the possible solar calibration angular range for the entire mission. The yaw maneuvers are performed in consecutive orbits to minimize change in instrument gains and SD BRF. The SD/SDSM screen vignetting function and SD BRF can be characterized from the relative response measured at different yaw angles.

Figure 2 shows examples of SD and SDSM solar screen vignetting functions characterized from yaw maneuver measurements. The absolute magnitude of the SD screen transmittance is computed as the mean detector response of screen-on and screen-off measurements. The absolute magnitude of SDSM screen is not required for calibration as SDSM measurements is relative, and the SDSM screen vignetting function is normalized to the mean.
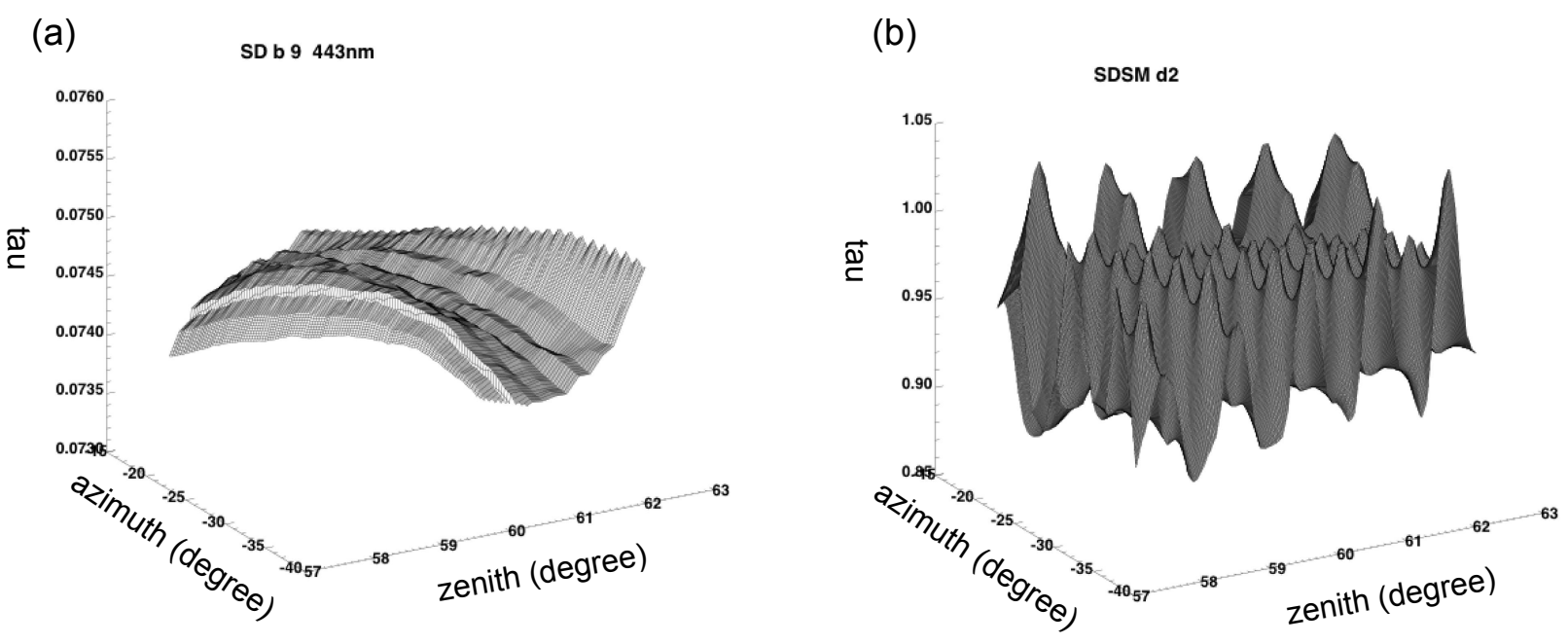

Figure 2. MODIS Aqua solar screen vignetting function (tau) for (a) SD band 9 and (b) SDSM detector 2 . The screen vignetting function are characterized by linear interpolation of yaw maneuver measurements. 
Figure 2 shows both SD and SDSM solar screens have pronounced features. The SD screen has a mean transmittance of $\sim 7.5 \%$ with variations of about $2 \%$. The SDSM screen has variations of about 10\%. Many studies have tried to address this issue as simple interpolation methods are not sufficient to produce well characterized screens due to coarse angular sampling collected during yaw maneuver $[4,5,6]$. In this paper, we will use screens produced from linear interpolation for simplicity. To mitigate the deficiency in screen characterization impact on the calibration, coherent noise correction is used to remove random noise and a SD degradation model is used to fine tune SDSM estimates for SD degradation at Near Infrared (NIR) and Short-Wave Infrared (SWIR).

The SD BRF changes smoothly at the measured angular range. The SD BRF is characterized by $2^{\text {nd }}$ order polynomial fits over the yaw maneuver measurements along zenith and azimuth angles. The fitted BRF is then normalized to the mean of prelaunch measured SD BRF to set the absolute SD reflectivity. Figure 3 shows the SD BRF is slightly different between the instrument view and the SDSM view angles at similar wavelengths.
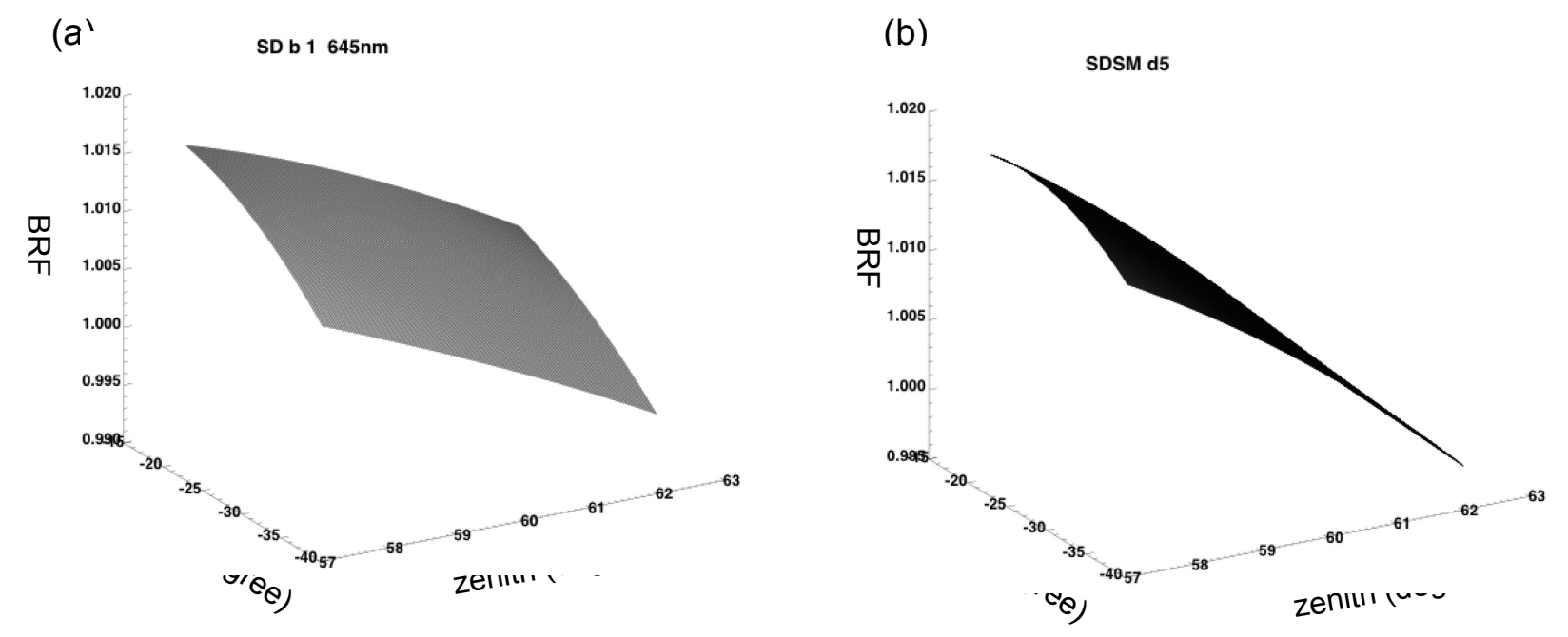

Figure. 3 MODIS Aqua SD BRF viewing from the instrument (a) and the SDSM (b). (a) shows BRF of band 1 and (b) shows BRF of SDSM detector 5 (646 nm).

The SD screen vignetting function and SD BRF at instrument view are characterized for all land 
bands (b1-b7, b17-b19 and b26). For ocean bands (b8-b16), only SD screen vignetting function is characterized as the SD radiances without screen attenuation saturate ocean bands. Note that the measured SD screen attenuation is actually the combined effects of screen transmission and SD BRF. The SDSM sun screen and SD BRF at SDSM view are characterized for each SDSM detectors. The SDSM measures changes in SD at 9 wavelengths, which are interpolated to instrument band's bandpass for the solar calibration of the RSB.

To facilitate the calibration process, four LUTs are created based on the characterization of SD/SDSM sun screens and the SD BRF at instrument and SDSM views. Each LUT is generated with 0.04 degree zenith and 0.1 degree azimuth angle interval. The range of the LUTs are from 57.5 to 62.5 degree zenith and -36 to -18.5 degree azimuth angles.

\section{SD degradation}

The SD degradation is described by the $\mathrm{H}$ factor, which is the ratio of instantaneous SD reflectivity to the reflectivity of the pristine diffuser [7]. The SDSM measures relative SD reflectivity as the ratio of sun view and SD view response (eq. 1),

$$
H_{m}(d, t)=\frac{\operatorname{tau}_{S D S M}(d, t, \theta, \gamma) * D C_{S D}(d, t)}{\cos \theta * B R F_{S D S} M_{S D}(d, t, \theta, \gamma) * D C_{S u n}(d, t)}
$$

where $H_{m}$ is the measured $\mathrm{H}$ factor, $D C_{S D}$ and $D C_{S u n}$ are the background subtracted SDSM detectors' voltages at SD and sun views, $\operatorname{tau}_{S D S M}$ is the SDSM solar screen transmission function, $B R F_{S D S M} S D$ is the SD BRF at SDSM view, $\theta, \gamma$ are the solar zenith and azimuth angles, $d$ is the SDSM detector number and $t$ is the time from first measurement.

Since SDSM measurements are relative, $H_{m}$ in eq. 1 is normalized to first measurement $\left(H_{m}\right.$ at $\mathrm{t}=0$ ) to represent the relative SD degradation from first SDSM measurement.

$$
H_{m}(d, t)=H_{m}(d, t) / H_{m}(d, 0)
$$


(a)

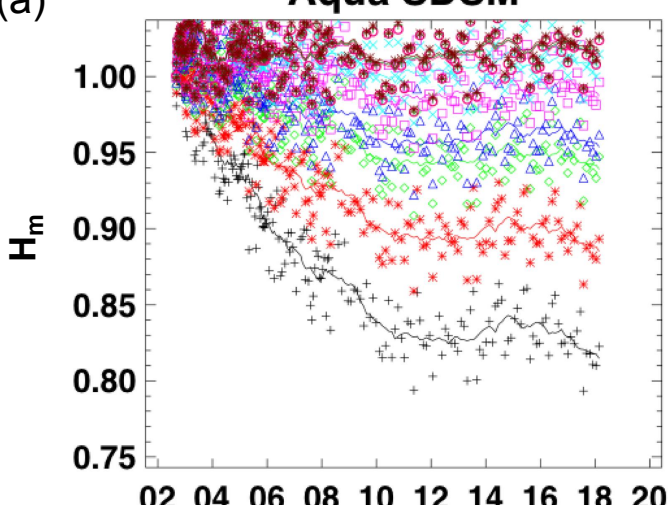

$02 \quad 0406 \quad 08101214161820$ (b)

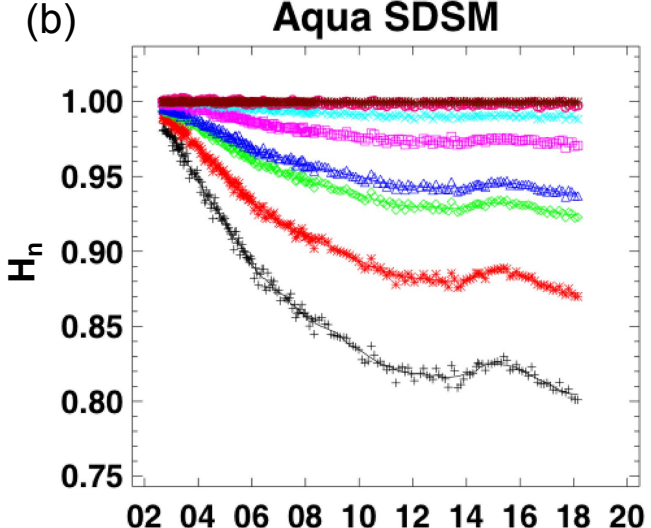

$+412 \mathrm{~nm} * 465 \mathrm{~nm} \quad \diamond 529 \mathrm{~nm} \quad \Delta 553 \mathrm{~nm} \quad \square 646 \mathrm{~nm} \times 746 \mathrm{~nm} \quad \circ 856 \mathrm{~nm}+904 \mathrm{~nm} \quad * 936 \mathrm{~nm}$

Figure. 4 MODIS Aqua SD degradation determined from SDSM measurements. (a) $H_{m}$, the measured ratio of the SDSM sun and SD view response. (b) $H_{n}$, normalized $H_{m}$ to remove coherent noise from SDSM screen and SD BRF uncertainties. The symbols are the measured values, and the curves are the 360 days running average.

Figure 4 a shows the time series of $H_{m}$ have very large uncertainties. The uncertainties are caused by the fine structures in the SDSM sun screen vignetting function that cannot be resolved by the coarse angular spacing in yaw maneuver data $[5,8]$. One way to reduce uncertainty is to assume the transmission of the pinhole screen to be wavelength independent and the uncertainties in SDSM measurements are coherent $[9,10]$. The coherent noise can be removed by normalizing $H_{m}$ to $H_{m}(9)$, the least degraded SD wavelength.

$$
H_{n}^{*}(d, t)=\frac{H_{m}(d, t)}{H_{m}(9, t)}
$$

Because the first Aqua SDSM measurements did not occur immediately after launch and the SD degradation is wavelength dependent, the bias between initial SDSM and prelaunch measurements will be wavelength dependent. To reduce spectral dependent biases, SD degradation prior to first SDSM measurement is approximated by extrapolating the $\mathrm{H}$ factor time series with the estimated solar exposure time prior to first SDSM measurement. In eq. $4, H_{n}$ is the normalized $\mathrm{H}$ factor $\left(H_{n}^{*}\right)$ adjusted by the SD degradation prior to first SDSM measurement $\left(\Delta H_{n}^{*}\right)$.

$$
H_{n}(d, t)=H_{n}^{*}(d, t)-\Delta H_{n}^{*}(d)
$$

Figure $4 \mathrm{~b}$ shows the bulk of the noise in $H_{m}$ (Figure $4 \mathrm{a}$ ) is removed in $H_{n}$ when $H_{m}(d, t)$ is 
normalized to $H_{m}(9, t)$. The $H_{n}$ time series shows the wavelength dependent adjustment for SD degradation prior to first SDSM measurement $\left(\Delta H_{n}^{*}\right)$. The $H_{n}$ still have residual uncertainties of up to $1 \%$. Since the change in SD reflectivity is expected to be gradual, the SD degradation is computed as the running average of $H_{n}$ (curve in Figure 4b) to reduce short term measurement noise. In Figure $4 \mathrm{~b}$, the $H_{n}(9, t)$ has no change overtime due to it being the normalizing SDSM detector. To compute the actual SD degradation, the $H_{n}$ needs to be adjusted by the SD degradation at the wavelength of SDSM detector 9.

\section{SD degradation model}

Previous studies have shown the degradation in SD reflectivity due to solar exposure is wavelength dependent $[2,3]$. The amount of degradation is found to be inversely proportional to the wavelength. We can describe the SD degradation with respective to wavelength in Eq. 5,

$$
D(\lambda, t)=D\left(\lambda_{r e f}, t\right) *\left(\frac{\lambda_{r e f}}{\lambda}\right)^{k(t)}
$$

where $D(\lambda, t)$ is the SD degradation at wavelength $\lambda$ and time $t, \lambda_{\text {ref }}$ is the reference wavelength. In eq. 5, $D$ is the inverse of $H(D=1-H)$ as $\mathrm{D}$ is the $\mathrm{SD}$ degradation and $\mathrm{H}$ is the SD reflectivity.

To solve eq. 5 , we set $\lambda_{\text {ref }}=936 \mathrm{~nm}$ (SDSM detector 9 wavelength) and compute initial $D$ from $H_{n}\left(D(\lambda, t)=1-H_{n}(\lambda, t)\right)$. The initial $k$ is set to its theoretical value of 4 [2]. We then solve $k$ by minimizing the RMS of the measured degradation $D(\lambda, t)$ and the modeled values $\left(D\left(\lambda_{\text {ref }}, t\right) *\right.$ $\left.\left(\frac{\lambda_{r e f}}{\lambda}\right)^{k(t)}\right)$. An iterative process is used to find the optimal solution. At first iteration, the $D(\lambda, t)$ is underestimated because $H_{n}(\lambda, t)$ assumes no degradation at detector 9 wavelength. In each iteration, the $D(\lambda, t)$ is recomputed by scaling them with the modeled SDSM detector 9 degradation $\left(D\left(\lambda_{\text {ref }}, t\right)\right)$. The iteration stops when the differences of modeled and measured SD degradation are under the specified threshold.

Previous study [3] had shown the SD degradation wavelength relationship might be less stable for wavelength shorter than $600 \mathrm{~nm}$. In this study, SDSM measurements from detector 4 to 9 (555 to $936 \mathrm{~nm}$, see table 1) were used to solve $k$ in eq. 5. Figure 5a shows the estimated SD degradation wavelength dependency $(k)$ is around 4 except a small dip in the first year of the mission. The averaged value $k$ of 3.98 is very close to the 4.03 reported for SNPP VIIRS [3], which uses the same type of SD. Figure $5 \mathrm{~b}$ shows the $\mathrm{H}$ factor predicted by SD degradation model fit and 
the SDSM measured $\mathrm{H}$ factor after factoring model estimated detector 9 degradation. Overall, the modeled $\mathrm{H}$ degradation matched well with measured $\mathrm{H}$, indicating the MODIS Aqua SD degradation has a wavelength dependency that can be well defined by eq. 5 .

(a)

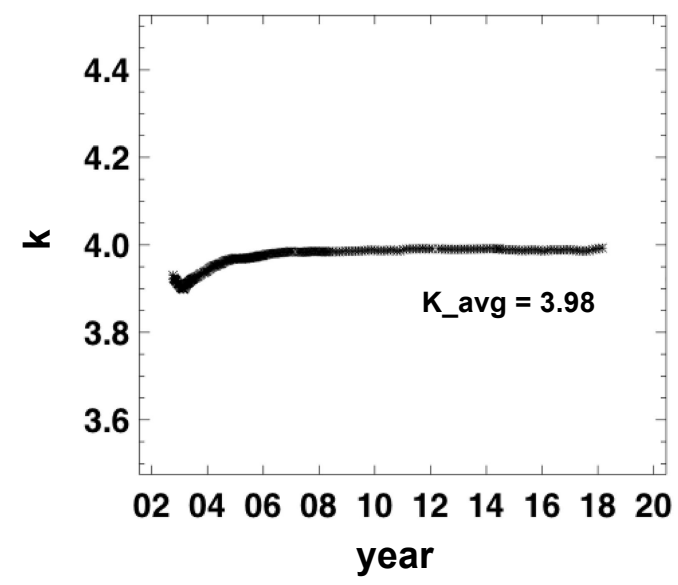

(b)

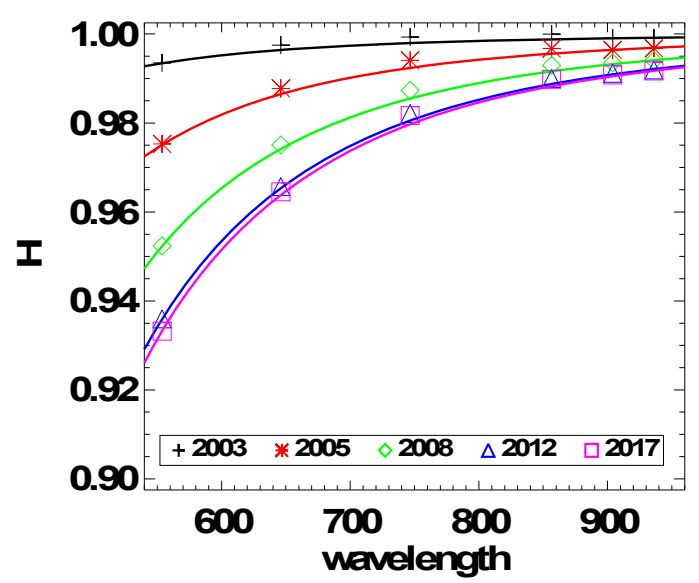

Figure 5. (a) Estimated MODIS Aqua SD degradation wavelength dependency $(k)$ over time. $\mathrm{K} \_a v g$ is the mean of $k$. (b) MODIS Aqua SD degradation measured (symbols) vs. wavelength model (curve) for selected years.

Figure 6a shows the solution for the SD degradation model at reference wavelength $\left(\lambda_{\text {ref }}\right.$ in eq. 5), which is equivalent to SDSM detector 9. The results indicate the SD should have degraded by $\sim 0.9 \%$ at $936 \mathrm{~nm}$ in the first 16 years of Aqua operation to maintain the degradation wavelength dependency measured by other SDSM detectors. Base on the model solution, we can compute the relative SD reflectivity at SDSM detector $9(H(9, t)$ in eq. 6$)$ and used to remove SDSM detector 9 biases in $H_{n}$ to complete the SD degradation ( $H$ in eq. 7 ) computation.

$$
\begin{aligned}
& H(9, t)=(1-D(936, t)) \\
& H(d, t)=H_{n}(d, t) * H(9, t)
\end{aligned}
$$

Figure $6 \mathrm{~b}$ shows the time series of final SD reflectivity computed from SDSM measurements. The $\mathrm{H}$ factor is computed from smoothed $H_{n}$ to remove coherent and random noises in $H_{m}$ and scaled by SDSM detector 9 degradation estimated from SD wavelength model. 

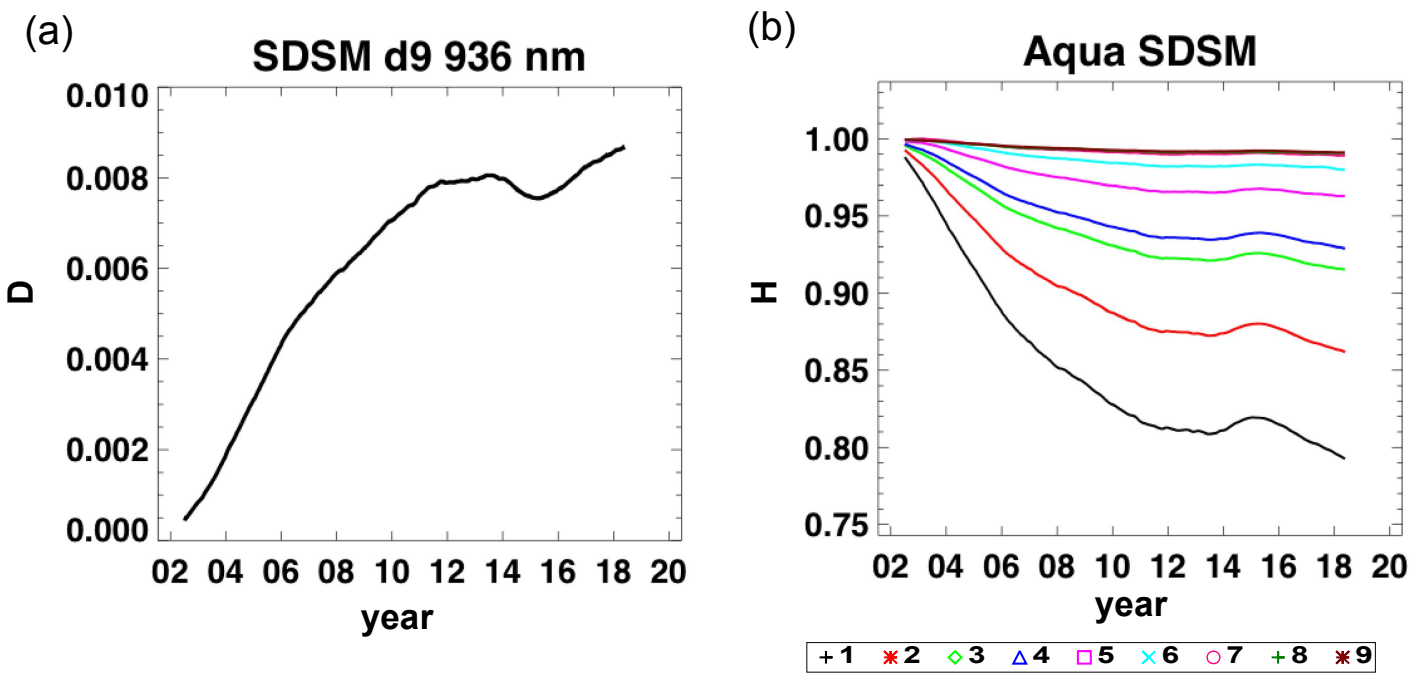

Figure 6. (a) SD degradation at SDSM detector 9. (b) SD reflectivity (H) for SDSM detectors 1 to 9. The $\mathrm{H}$ factor is derived from smoothed $H_{n}$ in Figure $4 \mathrm{~b}$ after scaling with the modeled SD reflectivity at SDSM detector 9.

The SD wavelength model can be used to estimate the SD degradation in wavelength longer than $936 \mathrm{~nm}$, the longest wavelength tracked by SDSM [3]. We can extend the model solution derived from 555 to $936 \mathrm{~nm}$ to approximate SD reflectivity at MODIS short-wave bands (band 5 to band 7) wavelengths (Figure 7). The predicted SD reflectivity changes for band 5 is $\sim 0.3 \%$ and less than $0.1 \%$ for bands 6 and 7 for the first 16 years of Aqua operation. The model estimated $\mathrm{H}$ factor should slightly improve short-wave bands calibration when compared with calibration performed with an assumption of no SD degradation beyond $936 \mathrm{~nm}$. 


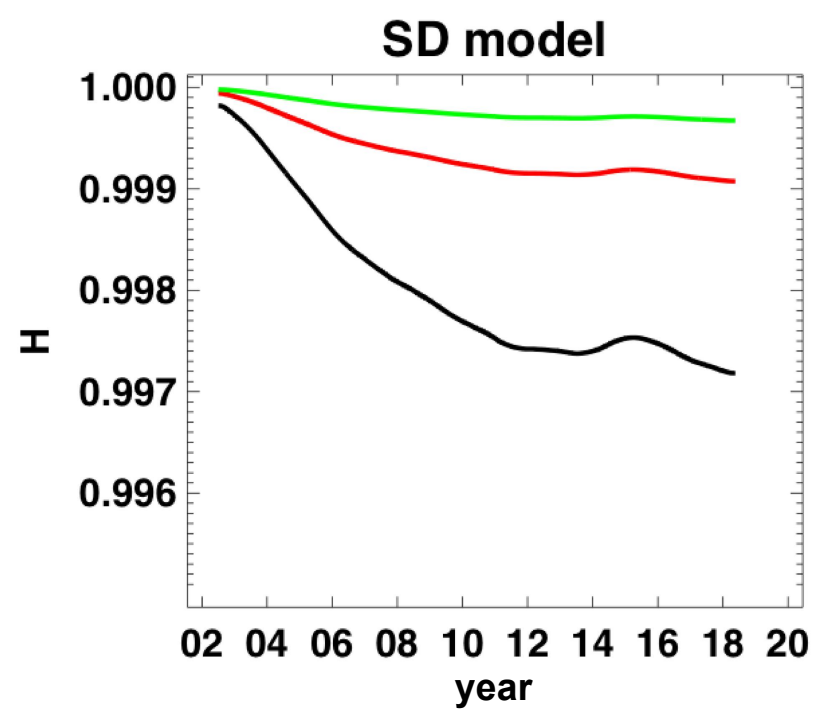

Figure 7. SD wavelength model estimated SD degradation at MODIS bands 5 to 7 wavelengths.

The model estimated degradation wavelength dependency can also be used to improve interpolation from SDSM detector wavelength to MODIS bands. For MODIS bands that don't have matching MODIS SDSM wavelength, SD degradation is computed by interpolation from 2 closest measured wavelengths. Without knowledge of SD degradation wavelength dependency, linear interpolation is often used. Using SD model (eq. 5), we can improve SD degradation interpolation by the model estimated degradation wavelength dependency. Figure 8 shows the time series of $\mathrm{H}$ factor differences between model and linear interpolation. The magnitude of biases is related to the total SD degradation and how far away is the band center wavelength from the SDSM detectors. Therefore, the difference between model and linear interpolation increases overtime due to increasing SD degradation. Bands $9(443 \mathrm{~nm})$ and $10(488 \mathrm{~nm})$ show the largest differences $(\sim 0.4$ and $0.3 \%$ over the first 16 years of operation) because SD degradation is large and the band centers are far away from the nearest SDSM detectors. 


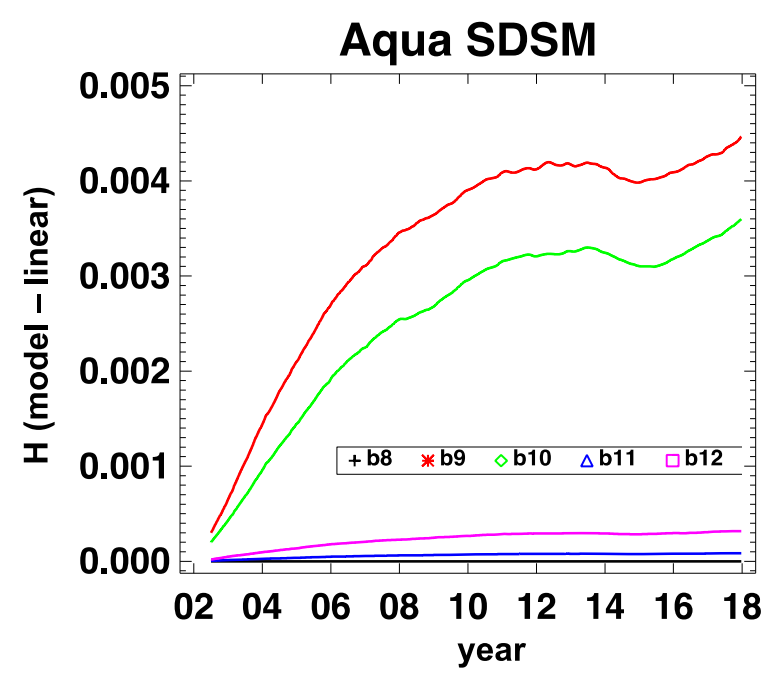

Figure 8. SD degradation at band center wavelength computed using linear and wavelength model interpolation.

\section{Summary}

In this paper, we presented a method to estimate the MODIS Aqua SD degradation to reduce the uncertainties in screen characterization and improve estimates in SD degradation in NIR and SWIR by using a SD degradation model. The large noise in SDSM measured SD degradation factors is coherent and can be minimized by normalizing to the least degraded SDSM detector 9. Based on the noise corrected SD degradation factors, the SD degradation wavelength dependency can be determined. The SD degradation wavelength dependency is computed using measurements from SDSM detector $4(555 \mathrm{~nm})$ to detector $8(904 \mathrm{~nm})$. Based on the SD degradation wavelength dependency, we can estimate SD degradation at SDSM detector 9 and SWIR bands. The SDSM detector 9 degradation is estimated at $\sim 0.9 \%$ over the first 16 years of Aqua operation. For SWIR bands, the SD degraded $\sim 0.3 \%$ at $1240 \mathrm{~nm}$ and less than $0.1 \%$ at $1640 \mathrm{~nm}$ and $2130 \mathrm{~nm}$.

The SD degradation wavelength dependency can also be used to improve the interpolation of the SD degradation from SDSM detector wavelengths to RSB wavelengths. The SD degradation at the wavelengths of bands 9 and 10 shows the largest difference between linear and model interpolated values. The differences are $\sim 0.4 \%$ and $\sim 0.3 \%$ for bands 9 and 10 for the first 16 years of operation.

This paper applied a method for SDSM calibration to determine SD degradation for MODIS 
RSB calibration. The use of coherent noise correction and the SD degradation model should improve the overall quality of the SD degradation temporal trends for RSB calibration. The method is also simple enough to be streamlined for use in operational calibration. The results have been applied in the Ocean Color Reprocessing R2018 of MODIS Aqua ocean color products [11].

\section{References:}

[1] W. Barnes, T. Pagano, and V. Salomonson, "Perlaunch Characteristics of the MODIS Resiolution Imaging Spectroradiometrer (MODIS) on EOS-AM1”, IEEE TGRS, vol. 36(4), 1088-1100 (1998).

[2] X. Shao, C. Cao, and T. Liu, "Spectral Dependent Degradation of the Solar Diffuser on SuomiNPP VIIRS Due to Surface Roughness-Induced Rayleigh Scattering", Remote Sensing, 254 (8), (2016). DOI: 10/3390/rs8030254

[3] N. Lei, and X. Xiong, "Suomi NPP VIIRS Solar Diffuser BRDF Degradation Factor at ShortWave Infrared Band Wavelengths", IEEE TGRS, 54 (10), (2016). DOI:

10/1109/TGRS.2016.2583220

[4] Z. Wang, and X. Xiong, "Characterization of MODIS SD screen vignetting function using observation from spacecraft yaw maneuvers", Proceedings of SPIE, vol. 7452, 745217-1 (2009).

[5] J. Sun, X. Xiong, and W. Barnes, "MODIS Solar Diffuser Stability Monitor Sun View Modeling", IEEE TGRS, vol. 43 (8), 1845-1854 (2015). DOI: 10.1109/TGRS.2005.852710

[6] E. Waluschka, X. Xiong, D. Myer, B. Guenther, W. Barnes, "MODIS solar calibration transmission screen modeling", Proceedings of SPIE, vol. 5652, 189-197 (2004).

[7] X. Xiong, J. Sun, J. Esposito and W. Barnes, "MODIS Reflective Solar Bands Calibration Algorithm and On-Orbit Performance", Proceedings of SPIE, vol. 4891, (2003). DOI:

$10.1117 / 12.466096$

[8] H. Chen, and X. Xiong, "MODIS solar diffuser stability monitor: function and application", Proceedings of SPIE, vol. 7452, 745218-1 (2009).

[9] R. Eplee, G. Meister, F. Patt, and C. Mcclain, "Uncertainty Assessment of the SeaWiFS On-Orbit Calibration", Proceedings of SPIE, vol. 8153, (2011). DOI: 10/1117/12.892340

[10] R. Eplee, K. Turpie, G. Meister, F. Patt, G. Fireman, B. Franz and C. Mcclain, "A Synthesis of VIIRS Solar and Lunar Calibration”, Proceedings of SPIE, vol. 8866, (2013). DOI:

$10 / 1117 / 12.2024069$

[11] https://oceancolor.gsfc.nasa.gov/reprocessing/r2018/aqua/. 